\title{
BMJ Open Trends in disability of instrumental activities of daily living among older Chinese adults, 1997-2006: population based study
}

\author{
Yajun Liang, ${ }^{1,2}$ Anna-Karin Welmer, ${ }^{2,3}$ Jette Möller, ${ }^{1}$ Chengxuan Qiu $^{2}$
}

To cite: Liang $\mathrm{Y}$, Welmer A-K, Möller J, et al. Trends in disability of instrumental activities of daily living among older Chinese adults, 1997-2006: population based study. BMJ Open 2017;7:e016996. doi:10.1136/ bmjopen-2017-016996

- Prepublication history and additional material for this paper are available online. To view please visit the journal (http:// dx.doi.org/10.1136/bmjopen2016-016996).

Received 24 March 2017

Revised 15 June 2017

Accepted 19 July 2017

\section{(a) CrossMark}

${ }^{1}$ Department of Public Health Sciences, Karolinska Institutet, Stockholm, Sweden

${ }^{2}$ Aging Research Center, Department of Neurobiology, Care Sciences and Society, Karolinska Institutet-Stockholm University, Stockholm, Sweden

${ }^{3}$ Functional Area Occupational Therapy \& Physiotherapy, Karolinska University Hospital, Stockholm, Sweden

Correspondence to Dr Yajun Liang; yajun.liang@ki.se

\section{ABSTRACT}

Background Data on trends for disability in instrumental activity of daily living (IADL) are sparse in older Chinese adults.

Objectives To assess trends in prevalence and incidence of IADL disability among older Chinese adults and to explore contributing factors.

Design Population based study.

Setting 15 provinces and municipalities in China. Subjects Participants $(a g e \geq 60)$ were from four waves of the China Health and Nutrition Survey, conducted in $1997(n=1533), 2000(n=1581), 2004(n=2028)$ and 2006 $(n=2256)$, and from two cohorts constructed within the national survey: cohort 1997-2004 $(n=712)$ and cohort 2000-2006 ( $n=823)$.

Measurements IADL disability was defined as inability to perform one or more of the following: shopping, cooking, using transportation, financing and telephoning. Data were analysed with logistic regression and generalised estimating equation models.

Results The prevalence of IADL disability significantly decreased from 1997 to 2006 in the total sample and in all of the subgroups by age, sex, living region and IADL items (all $p_{\text {trend }}<0.05$ ). The incidence of IADL disability remained stable from cohort 1997-2004 to cohort 2000-2006 in the total sample and in all of the subgroups (all $p>0.10$ ). The recovery rate from IADL disability significantly increased over time in those aged $60-69$ years $(p=0.03)$. Living in a rural area or access to local clinics for healthcare was less disabling over time $\left(\mathrm{p}_{\text {trend }}<0.02\right)$.

Conclusions The prevalence of IADL disability decreased among older Chinese adults during 1997-2006, whereas the incidence remained stable. The declining prevalence of IADL disability might be partly due to the decreased duration of IADL disability, and to improvements in living conditions and healthcare facilities over time.

\section{INTRODUCTION}

Functional ability deteriorates progressively as people age. Given the significant impact of functional dependence on quality of life and the social care system, ${ }^{1}$ clarifying time trends in physical disability as well as exploring modifiable risk factors for disability will have important implications for public health and
Strengths and limitations of this study

- The study had a nationwide sample with high participation rates, and the data were collected using a consistent design and approach over time.

- Certain items in instrumental activity of daily living (IADL) (eg, housekeeping and handling medications) were not available in the dataset, which might slightly affect the estimates of the prevalence and incidence of IADL disability.

- We were not able to explore the effects of other sociocultural and environmental factors and additional chronic diseases due to lack of specific data.

- The use of self-reported information on lifestyle factors and chronic diseases might lead to underestimation of their prevalence and potentially biased associations with disability.

- For the trends in incidence and recovery rates of IADL disability, participants in the two cohorts differed from those who were lost to follow-up with regard to age, education and marital status, which might affect the internal validity of the findings.

- Finally, because our study participants were relatively young (mean age 69.4 years), caution is needed when the trend in IADL disability from our study is generalised to older people.

policy development. To date, most studies on disability trends have focused mainly on basic activities of daily living (ADLs), ${ }^{2-6}$ which measured self-care tasks of everyday life, including feeding, dressing, bathing, toileting, transferring and continence. However, as disability in basic ADL function represents a more severe stage of physical dysfunction, it is crucial to investigate trends in functional loss from an early stage. Instrumental ADLs (IADLs), which include higher level tasks, such as using the telephone, preparing meals, shopping, housekeeping, using transportation and managing finances, ${ }^{8}$ usually decline prior to impairment in basic ADLs. Thus assessment of IADLs may identify 
Table 1 Characteristics of participants in the surveys in 1997, 2000, 2004 and 2006

\begin{tabular}{|c|c|c|c|c|c|}
\hline Characteristic* $^{*}$ & $1997(n=1533)$ & $2000(n=1581)$ & $2004(n=2028)$ & $2006(n=2256)$ & $p_{\text {trend }} t$ \\
\hline Age (years) & $69.0(7.0)$ & $69.2(6.7)$ & $69.5(6.8)$ & $69.6(7.0)$ & 0.007 \\
\hline Women & $820(53.5)$ & $853(54.0)$ & 1067 (52.6) & $1189(52.7)$ & 0.410 \\
\hline No formal school & $993(70.0)$ & $892(61.1)$ & $1004(49.7)$ & $1204(53.5)$ & \\
\hline Primary school & $218(15.4)$ & $275(18.8)$ & $501(24.8)$ & $429(19.1)$ & \\
\hline \multicolumn{6}{|l|}{ Living region } \\
\hline Urban & $640(41.7)$ & $637(40.3)$ & 795 (39.2) & $848(37.6)$ & \\
\hline Rural & $893(58.3)$ & $944(59.7)$ & $1233(60.8)$ & $1408(62.4)$ & 0.002 \\
\hline \multicolumn{6}{|l|}{ Race } \\
\hline Han majority & $1357(91.0)$ & $1404(89.4)$ & $1801(88.8)$ & $1979(87.7)$ & \\
\hline \multicolumn{6}{|l|}{ Access to healthcare facility } \\
\hline Hospitals & $363(35.1)$ & $346(32.2)$ & $367(27.8)$ & $396(28.4)$ & \\
\hline Local clinics & $671(64.9)$ & $729(67.8)$ & $952(72.2)$ & 997 (71.6) & $<0.001$ \\
\hline Ever smoking & $444(29.1)$ & $450(28.6)$ & $686(33.9)$ & $743(33.0)$ & $<0.001$ \\
\hline Alcohol intake & $242(16.1)$ & $282(18.4)$ & 335 (16.5) & $363(16.1)$ & 0.796 \\
\hline Physical inactivity & 1365 (89.0) & 1469 (92.9) & 1903 (93.8) & 2151 (95.3) & $<0.001$ \\
\hline Unfavourable diet & $1038(69.2)$ & $1102(70.4)$ & 1352 (67.2) & 1555 (70.0) & 0.529 \\
\hline \multicolumn{6}{|l|}{ Chronic multimorbidity } \\
\hline No & 1326 (86.5) & 1300 (82.2) & 1493 (73.6) & 1702 (75.4) & \\
\hline
\end{tabular}

Values are mean (SD) for age and $\mathrm{n}(\%)$ for other variables.

*The number of subjects with missing values was 250 for education, 52 for race, 110 for marital status, 2577 for healthcare facility, 19 for smoking, 84 for alcohol intake and 99 for diet. When these factors were considered as covariates in subsequent analyses, a dummy variable for each of these factors was created to represent those with the missing value.

†The linear trend was tested using separate generalised estimation equation regression models if applicable, controlling for age, sex, education, living region, race and marital status.

incipient decline in self-care ADLs among older adults who might otherwise appear capable and healthy. ${ }^{9}$ Furthermore, IADLs require higher neuropsychological functioning, which can be severely affected in people with mild cognitive impairment. ${ }^{10}{ }^{11}$ This further supports the importance of studies on time trend in IADL dysfunction.

However, time trends for IADL disability in older adults have not been well studied. In the USA, the prevalence of IADL disability was stable among older adults during the period 1998-2008. ${ }^{12}$ In Europe, the prevalence of IADL disability either decreased (eg, among men in Italy and The Netherlands, and among women in Denmark, France and Sweden) or remained stable among older adults aged $\geq 65$ years in other European countries for the period 2004-2013. ${ }^{13}$ We have previously reported that both prevalence and incidence of ADL disability had declined during the period 1993-2006 among older Chinese adults. ${ }^{45}$ Because ADLs and IADLs measure different aspects of functional capacity, trends for IADL disability might differ from those of self-care ADL disability. However, reports from nationwide surveys on trends in IADL disability among older Chinese adults are lacking.

Late life functional disability has been associated with demographic factors (eg, being unmarried, illiterate and not living in an urban area), ${ }^{14}$ unhealthy lifestyle (eg, smoking), ${ }^{15}$ vascular risk factors (eg, obesity, hypertension and diabetes) ${ }_{16}^{16}$ chronic diseases (eg, stroke, dementia and arthritis) ${ }^{18}$ and geriatric syndromes (eg, depressive symptoms, dizziness, falls, hearing or visual impairment, osteoporosis and sleep disturbance). ${ }^{19}$ Therefore, the time trends observed for disability might be attributable to the variations in these influential factors. For example, data from The Netherlands indicate an increase in prevalence of chronic diseases but a relatively stable prevalence of functional disability, suggesting that chronic diseases might have become less disabling. ${ }^{20}$ Similarly, stroke and multiple cardiometabolic diseases were found to be less disabling in ADLs over time in China. ${ }^{4}$ However, the impact of these factors on IADL disability has not yet been investigated among older Chinese adults. Further 
Table 2 Trends for the prevalence of instrumental activity of daily living disability over time in 1997-2006 by age, gender, living region and specific instrumental activity of daily living items

\begin{tabular}{|c|c|c|c|c|c|}
\hline IADL disability & $1997(n=1533)$ & $2000(n=1581)$ & $2004(n=2028)$ & $2006(n=2256)$ & $\mathbf{p}_{\text {trend }}{ }^{*}$ \\
\hline Total sample & 597 (38.9) & $562(35.5)$ & 554 (27.3) & $601(26.6)$ & $<0.001$ \\
\hline \multicolumn{6}{|l|}{ Age (years) } \\
\hline $60-69$ & $266(27.5)$ & 231 (24.3) & $181(15.3)$ & $180(13.8)$ & $<0.001$ \\
\hline $70-79$ & $209(48.7)$ & $233(46.0)$ & 255 (37.9) & $282(37.5)$ & $<0.001$ \\
\hline$\geq 80$ & $122(88.4)$ & 98 (79.0) & $118(69.0)$ & 139 (68.5) & 0.002 \\
\hline \multicolumn{6}{|l|}{ Gender } \\
\hline Men & 213 (29.9) & $174(23.9)$ & $183(19.0)$ & 217 (20.3) & $<0.001$ \\
\hline Women & $384(46.8)$ & $388(45.5)$ & $371(34.8)$ & $384(32.3)$ & $<0.001$ \\
\hline \multicolumn{6}{|l|}{ Living region } \\
\hline Urban & $202(31.6)$ & $159(25.0)$ & $168(21.1)$ & $194(22.9)$ & $<0.001$ \\
\hline Rural & $395(44.2)$ & $403(42.7)$ & $386(31.3)$ & 407 (28.9) & $<0.001$ \\
\hline \multicolumn{6}{|l|}{ Specific IADL items } \\
\hline Shopping & $150(9.8)$ & $140(8.9)$ & $185(9.2)$ & $221(9.8)$ & 0.047 \\
\hline Cooking & $157(10.3)$ & $119(7.5)$ & $173(8.5)$ & $178(7.9)$ & $<0.001$ \\
\hline Transportation & $421(27.8)$ & $391(24.8)$ & $410(20.3)$ & $479(21.4)$ & $<0.001$ \\
\hline Managing money & $167(11.0)$ & $136(8.6)$ & $160(7.9)$ & $200(8.9)$ & $<0.001$ \\
\hline Using telephone & $355(24.2)$ & $336(21.8)$ & $281(14.1)$ & $290(13.0)$ & $<0.001$ \\
\hline
\end{tabular}

*The generalised estimation equation model was performed after controlling for age, sex, education, living region, race, marital status, access to healthcare facility, ever smoking, alcohol intake, leisure activity and chronic multimorbidity.

IADL, instrumental activity of daily living.

research on how chronic health conditions and physical disability are associated over time is needed.

We hypothesised that together with the decreasing prevalence and incidence of disability in ADLs, the incidence and prevalence of IADL disability might also have decreased during the same time period in older Chinese adults. In this study, we investigated the secular trends in both prevalence and incidence of IADL disability among older Chinese adults, and also explored factors that may potentially contribute to the trends in IADL disability.

\section{METHODS}

\section{Study design and population}

Study participants were derived from the China Health and Nutrition Survey (CHNS), which is an ongoing longitudinal survey on health risk factors, nutritional status and health outcomes in Chinese populations nationwide (age $\geq 2$ years). The study sample for CHNS was drawn from 15 provinces and municipalities through a multistage, randomised, cluster sampling process, as described in detail elsewhere. ${ }^{21}$ Counties in these provinces were stratified by income (low, middle and high), and a weighted sampling scheme was used to randomly select four counties in each province. Surveys for CHNS were conducted in 1989, 1991, 1993, 1997, 2000, 2004, 2006, 2009 and 2011, covering household, community and health/family planning facility data. Follow-up levels were high, but families who migrated from one community to a new one were not followed. Since 1997, new households in the original communities were added to replace households no longer participating in the study. In this study, variables for IADLs were assessed only in surveys in 1997, 2000, 2004 and 2006, and the trend in the prevalence of IADL disability was assessed based on these four waves of cross sectional surveys.

We included participants who were aged $\geq 60$ years and who had been assessed with IADLs: 1533 (participation rate, $88.3 \%)$ in 1997, 1581 (85.9\%) in 2000, 2028 (92.6\%) in 2004 and $2256(94.0 \%)$ in 2006. Among these participants, $37.1 \%, 31.4 \%, 19.1 \%$ and $12.3 \%$ participated in 1 , 2, 3 and all 4 waves of the survey, respectively. Compared with people who participated in only one wave, those who participated in two or more waves of the survey were younger, were more likely to be married, had a lower level of education, were more likely to drink alcohol and participate in physical activity, and were less likely to have chronic diseases, but no significant difference was found in the distribution of gender, race, living region, access to health facility, smoking or diet.

In addition, to explore changes over time in the incidence of IADL disability, we identified two consecutive cohorts within the four waves of the CHNS (cohort 1 (1997-2004) and cohort 2 (2000-2006)), as previously described. ${ }^{5}$ This was possible because some participants in CHNS were selected to participate in more than one wave of the survey. Specifically, cohort 1 (1997-2004) included participants who were free of IADL disability in 1997 and who were followed in 2000 or 2004 or both 
Table 3 Incidence rate of instrumental activity of daily living disability by age, sex, living region and specific instrumental activity of daily living items in 1997-2004 and 2000-2006

\begin{tabular}{|c|c|c|c|c|c|c|c|c|c|}
\hline \multirow[b]{2}{*}{ IADL disability } & \multicolumn{4}{|c|}{ Cohort 1997-2004 (n=712) } & \multicolumn{4}{|c|}{ Cohort 2000-2006 (n=823) } & \multirow[b]{2}{*}{$\mathbf{p}^{*}$} \\
\hline & $\begin{array}{l}\text { No of } \\
\text { subjects }\end{array}$ & $\begin{array}{l}\text { No of } \\
\text { cases }\end{array}$ & $\begin{array}{l}\text { Person } \\
\text { years }\end{array}$ & $\begin{array}{l}\text { Incidence rate, } \\
\text { per } 1000 \text { person } \\
\text { years }\end{array}$ & $\begin{array}{l}\text { No of } \\
\text { subjects }\end{array}$ & $\begin{array}{l}\text { No of } \\
\text { cases }\end{array}$ & $\begin{array}{l}\text { Person } \\
\text { years }\end{array}$ & $\begin{array}{l}\text { Incidence } \\
\text { rate, per } 1000 \\
\text { person years }\end{array}$ & \\
\hline Total sample & 712 & 231 & 3283 & 70.4 & 823 & 227 & 3818 & 59.5 & 0.110 \\
\hline \multicolumn{10}{|l|}{ Age (years) } \\
\hline $60-69$ & 559 & 157 & 2672 & 58.8 & 612 & 139 & 2952 & 47.1 & 0.303 \\
\hline$\geq 70$ & 153 & 74 & 611 & 121.1 & 211 & 88 & 866 & 101.6 & 0.640 \\
\hline \multicolumn{10}{|l|}{ Gender } \\
\hline Men & 380 & 98 & 1887 & 51.9 & 446 & 112 & 2131 & 52.6 & 0.603 \\
\hline Women & 332 & 133 & 1395 & 95.3 & 377 & 115 & 1687 & 68.2 & 0.124 \\
\hline \multicolumn{10}{|l|}{ Living region } \\
\hline Urban & 325 & 80 & 1639 & 48.8 & 381 & 95 & 1823 & 52.1 & 0.695 \\
\hline Rural & 387 & 151 & 1644 & 91.8 & 442 & 132 & 1995 & 66.2 & 0.287 \\
\hline \multicolumn{10}{|l|}{ Specific IADLs } \\
\hline Shopping & 712 & 69 & 3283 & 21.0 & 823 & 86 & 3818 & 22.5 & 0.421 \\
\hline Cooking & 712 & 63 & 3283 & 19.2 & 823 & 78 & 3818 & 20.4 & 0.707 \\
\hline Transportation & 712 & 169 & 3283 & 51.5 & 823 & 182 & 3818 & 47.7 & 0.109 \\
\hline $\begin{array}{l}\text { Managing } \\
\text { money }\end{array}$ & 712 & 60 & 3283 & 18.3 & 823 & 76 & 3818 & 19.9 & 0.252 \\
\hline $\begin{array}{l}\text { Using } \\
\text { telephone }\end{array}$ & 712 & 104 & 3283 & 31.7 & 823 & 104 & 3818 & 27.2 & 0.873 \\
\hline
\end{tabular}

*The generalised estimation equation model was performed to test the statistical difference in the incidence rates between cohort 1 (19972004) and cohort 2 (2000-2006) after controlling for age, sex, education, living region, race, marital status, access to healthcare facility, ever smoking, alcohol intake, leisure activity, chronic multimorbidity and follow-up time.

IADL, instrumental activity of daily living.

$(\mathrm{n}=712)$. Similarly, cohort 2 (2000-2006) included participants who were free of IADL disability in 2000 and who were followed in 2004 or 2006 or both $(n=823)$. In the two cohorts, compared with those who were lost to follow-ups (dropouts), persons who had at least one follow-up assessment were younger, had a lower level of education and were more likely to be married at baseline in cohort 1 ; baseline characteristics of participants were similar to those who were lost to follow-up except that participants were younger in cohort 2 (see online supplementary table 1).

Furthermore, we identified two cohorts of individuals with IADL disability within the CHNS to determine the recovery rate of IADL disability. The cohort 1997-2004 included individuals who were disabled in terms of IADLs in 1997 and who were followed in 2000 or 2004 or both $(\mathrm{n}=390)$, and the cohort 2000-2006 included those who had IADL disability in 2000 and who were followed in 2004 or 2006 or both $(\mathrm{n}=377)$.

\section{Data collection and definitions}

Data on demographic factors, healthcare, lifestyle and chronic diseases were collected by trained and certified health professionals through interviews and physical examinations. ${ }^{21}$ Education was divided into no formal school ( $<1$ year), primary school ( $1-6$ years) and middle school or above ( $>6$ years). Living region was divided into urban versus rural area (the area where most people work as farmers). Race was dichotomised into Han majority and non-Han minority.

Smoking status was categorised into never and ever (former and current) smoking. Alcohol intake was categorised into no and regular drinking (ie, regularly drinking alcoholic beverage $\geq 3$ times a week). ${ }^{4}$ Physically inactive was defined as weekly physical exercise less than the equivalent of $150 \mathrm{~min}$ of moderate intensity aerobic physical activity or $75 \mathrm{~min}$ vigorous intensity aerobic physical activity. ${ }^{22}$ According to the US recommendations on dietary reference intake for total energy, acceptable macronutrient distributions are $45-65 \%$ for carbohydrates, $20-35 \%$ for fat and $10-35 \%$ for protein. ${ }^{23}$ In this study, on the basis of a 3 day record of household meals, unfavourable diet was defined as having $\geq 1$ of three macronutrients out of the US dietary reference intake range. ${ }^{5}$

Chronic multimorbidity was defined as concurrently having $\geq 2$ chronic health conditions that included obesity (ie, body mass index $\left.\geq 28 \mathrm{~kg} / \mathrm{m}^{2}\right)^{24}$ and hypertension (systolic/diastolic pressure $\geq 140 / 90 \mathrm{~mm} \mathrm{Hg}$ or currently 


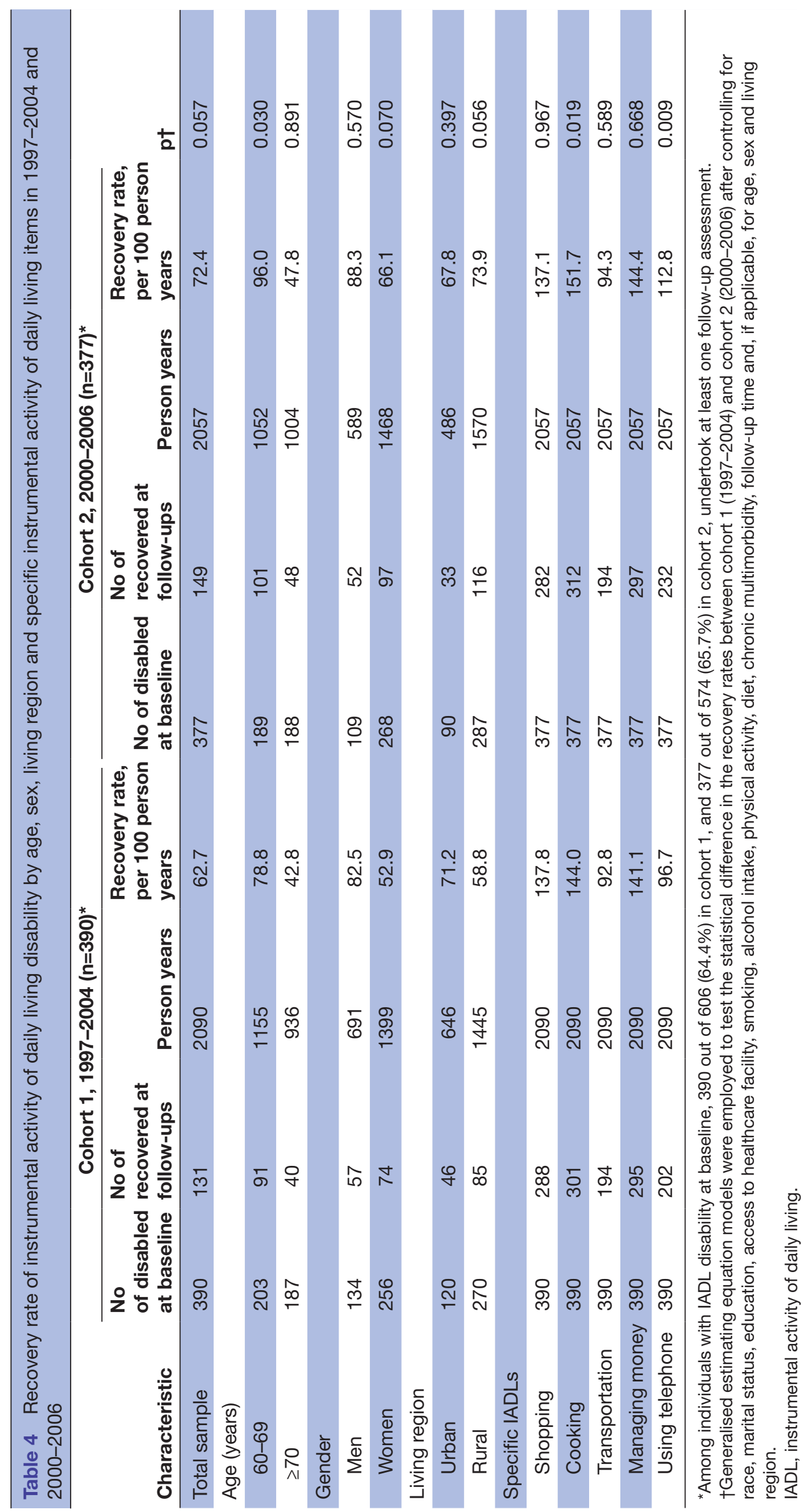




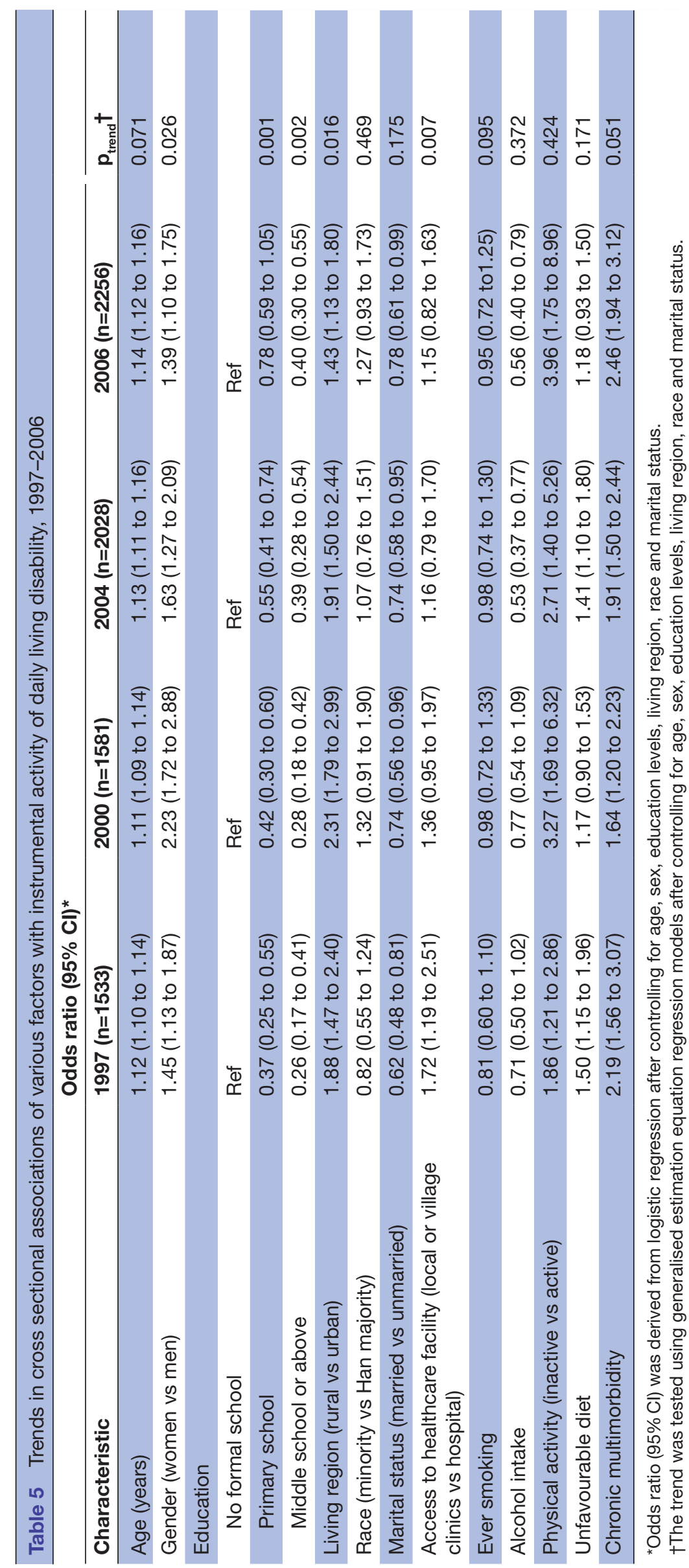


receiving antihypertensive medications ${ }^{25}$; self-reported physician diagnosis of diabetes, heart disease or myocardial infarction, and stroke; self-reported joint or muscle pain, eye/hearing problems, urinating or defecating problems, anxiety, exhaustion, unexplained weight loss, memory complaint and other chronic non-communicable conditions.

IADLs were measured according to self-reported responses to questions (ie, do you have any difficulty in doing this activity?), which involved five activities: shopping (buying food, clothes, etc), cooking, using public transportation, managing money (record income and expenses, etc) and using the telephone. Those who needed help or were unable to perform $\geq 1$ of these activities were defined as having IADL disability. For some IADL items (eg, cooking), participants were coded as independent if they stated able to do even if they never did. Those having IADL disability but no disability for ADL items (eg, transferring, dressing, toileting, bathing and feeding) were defined as having only IADL disability.

\section{Statistical analysis}

Trends in baseline characteristics across the four waves of the survey were assessed using the generalised estimating equation (GEE) models to account for the correlation of repeated participation of the same individuals (eg, the same persons included in two or more surveys). The incidence of IADL disability was calculated according to person years of follow-up. For persons who did not develop IADL disability, the follow-up time was calculated from the date of the baseline survey to the date of the last contact. For those who developed IADL disability, the onset time of IADL disability was assumed to be in the midpoint between the dates of the two surveys. ${ }^{5}$ Furthermore, we also assessed the recovery rate in those who had IADL disability at baseline. The follow-up time for the recovery rate was calculated in the same way as that for the incidence of IADL disability. GEE models were employed to assess the trends in prevalence, incidence and recovery rates of IADL disability over time after controlling for possible covariates (eg, age, sex, education, living region, race, marital status, access to healthcare facility, ever smoking, alcohol intake, leisure activity and chronic multimorbidity).

Logistic regression models were used to examine the cross sectional associations between potential determinants and IADL disability within each survey. The GEE model was used to assess the trends in strength of associations between various factors and IADL disability across surveys, in which an interaction term between year of assessment and individual factors was included into the model together with covariates.

To assess the impact of dropouts (attrition rate) during follow-ups on the trends in incidence and recovery of IADL disability, multiple imputation analysis was performed using the Sequential Regression Imputation
Method. Five multiple imputation datasets were generated and the pooled dataset was used for estimation of trends.

IBM SPSS Statistics 22 for Windows (IBM SPSS Inc, Chicago, Illinois, USA) was used for all analyses. A p for trend value was provided for assessing the trend in characteristics of the study participants and the prevalence of IADL disability. A $p$ value for two group comparison was provided for the incidence and recovery rate of IADL disability. Statistical significance was set at $p \leq 0.05$.

\section{RESULTS}

Over time, from the survey in 1997 to 2006, participants were older and more likely to be educated, live in a rural region, have a minority race, be married, smoke and have chronic multimorbidity (all $\mathrm{p}_{\text {trend }}<0.01$ ) but less likely to go to hospital (vs local clinic) for healthcare and participate in physical activity (both $\mathrm{p}_{\text {trend }}<0.001$ ). The proportions of women, alcohol intake and unfavourable diet did not differ significantly across surveys $\left(p_{\text {trend }}>0.40\right)$ (table 1).

After controlling for covariates, the prevalence of IADL disability significantly decreased in the total sample and in all subgroups by age, sex and living regions (all $\mathrm{p}_{\text {trend }}$ $<0.01)$. The prevalence of disability in all IADL items decreased over time (all $\mathrm{p}_{\text {trend }}<0.05$ ) (table 2). In addition, the declining trend in the prevalence of IADL disability was unchanged after excluding persons with ADL disability, except for the prevalence of disability in shopping or cooking, which was relatively stable (both $\mathrm{p}_{\text {trend }}>0.10$ ) (see online supplementary table 2 ).

The incidence of IADL disability was stable between 1997-2004 and 2000-2006 in the total sample, in all subgroups by age, sex and living regions, and in all IADL items (all p $>0.10$ ) (table 3). Furthermore, the multiple imputation analysis showed similar results in terms of the time trends in incidence of IADL disability (data not shown).

Among individuals who had IADL disability, the recovery rate from IADL disability marginally increased over time in the total sample $(\mathrm{p}=0.057)$ and significantly increased among those aged 60-69 years $(\mathrm{p}=0.03)$. For individual items of IADLs, the recovery rate significantly increased over time for disability in cooking $(\mathrm{p}=0.019)$ and using the telephone $(\mathrm{p}=0.009)$, whereas there was no change in the other three items (table 4). The multiple imputation analysis showed a similar trend in recovery rate of IADL disability (data not shown).

Logistic regression model showed that IADL disability was significantly associated with older age, female sex, low education, living in a rural area, being unmarried, being physically inactive and having multimorbidity at each survey, with local or village clinics (vs hospitals) in 1997, with regular alcohol intake in 2004 and 2006, and with unfavourable diet in 1997 and 2004. The GEE model showed that women were less likely to have IADL disability over time $\left(\mathrm{p}_{\text {trend }}=0.026\right)$; the protective effects of 
education on IADL disability decreased over time (both $\left.\mathrm{p}_{\text {trend }}<0.01\right)$; living in a rural area or having access to local or village clinics for healthcare services was less disabling over time $\left(\mathrm{p}_{\text {trend }}<0.02\right)$; however, chronic multimorbidity tended to become more disabling in IADLs across the surveys $\left(\mathrm{p}_{\text {trend }}=0.051\right)$ (table 5$)$.

\section{DISCUSSION}

Findings from this nationwide study add to the current literature with regard to the time trends in occurrence of IADL disability (prevalence, incidence and recovery rates) as well as the potential contributing factors to trends among older Chinese adults. Specifically, the prevalence of IADL disability among people aged $\geq 60$ years decreased from 1997 to 2006. The incidence of IADL disability was stable along with the improvement in recovery from IADL disability during the same period. The decreasing prevalence of IADL disability might be partly due to the less disabling effect of living in a rural area or having access to local clinics for healthcare services.

In line with the findings from the Shanghai region, ${ }^{26}$ our nationwide data showed a decreasing trend in prevalence of IADL disability among older Chinese adults during 1997-2006. Of note, while the incidence of IADL disability remained stable from cohort 1997-2004 to cohort 2000-2006, the recovery rate of IADL disability was increased (or duration of disability was decreased) during the same period. Therefore, the declining prevalence of IADL disability was partly attributable to the increase in recovery from IADL disability over time. Functional disability is a complex and dynamic process that involves not only biological and chronic conditions but also a social and environmental context. Older people may experience frequent transition from being independent to dependent or vice versa. Our study emphasises that recovery needs to be considered when examining the time trends in the prevalence of functional disability.

To the best of our knowledge, the secular trends in disability for specific IADL items have not been reported previously in older Chinese adults. We found a declining prevalence of disability in all specific IADL items, which was different from the previous findings from other countries. $^{27} 28$ The US Health and Retirement Study showed that the prevalence of disability decreased for shopping but did not change for other items (eg, managing money, preparing meals, using the phone and taking medication) during the period 1995-2004. ${ }^{27}$ A Swedish study of 75-year-olds showed a decrease in the prevalence of disability for cleaning and using transportation but not for shopping and cooking during the period 1976-2006. ${ }^{28}$ The inconsistent findings could be partly due to differences in the characteristics of the study populations (eg, age, education and ethnicity) and study settings. ${ }^{29}$

The trends in IADL disability are likely to be determined by multiple influential factors for IADL disability. Participants had higher levels of education over time, which might contribute to the declining prevalence of
IADL disability, although the effect of education tended to decrease over time. ${ }^{5}$ Furthermore, living in rural areas was associated with an increased risk of IADL disability, but its disabling effect decreased over time. Indeed, socioeconomic environment and living conditions in rural China have improved greatly during the study period, ${ }^{30}$ which might contribute to both the declining prevalence and increasing recovery of IADL disability. ${ }^{31}$ In addition, the less disabling effect of access to local clinics (vs hospitals) for healthcare might reflect the gradual improvement in healthcare service and basic infrastructure in rural China over time. ${ }^{30} 32$

Of note, chronic multimorbidity was found to be less disabling in basic self-care ADLs over time in earlier studies. ${ }^{40}$ There may be different explanations for this: first, improvement in living environment as well as an increase in the use of aids and devices to facilitate greater independence of older adults with chronic diseases; and second, advancements in medical and health services lead to early diagnosis and better treatment of chronic diseases (eg, stroke). ${ }^{33}$ However, our data showed that chronic multimorbidity appeared to have a more disabling impact on IADLs over time. As dependence in IADLs may indicate an early sign of functional decline in old age, intervention programmes targeting major modifiable risk factors for chronic diseases might help to reduce the risk of IADL disability and its progression to disability in self-care ADLs.

The major strength of this study was the nationwide sample derived from the large and diverse population in China. Moreover, data were collected using consistent approaches over time. In addition, the participation rates across the four waves of the survey were high (86-94\%). However, this study also had limitations. First, certain items in the IADL (eg, housekeeping and handling medications) were not available in the CHNS dataset, which might affect estimates of the prevalence and incidence of IADL disability. ${ }^{35}$ Second, we were not able to explore the effects of other sociocultural and environmental factors (eg, improved medical techniques, social and family support, improvements in housing and public transport) and additional chronic diseases (eg, chronic obstructive pulmonary disease and dementia) due to lack of specific data. Third, the use of self-reported information on lifestyle factors and chronic diseases might lead to underestimation of their prevalence and potentially biased associations with IADL disability. Fourth, participants in the two cohorts did differ from those who were lost to follow-up with regard to demographic features (eg, age, education and marital status), which might affect the internal validity of the findings. Finally, because our study participants were relatively young (mean age 69.4 years), caution is needed when the trend in IADL disability from our study is generalised to older people.

In summary, the prevalence of IADL disability among older Chinese adults decreased during the period 19972006, along with a stable incidence and an increased recovery rate from IADL disability. The favourable trend in prevalence of IADL disability might be partly 
attributable to the improved living environment, and to better healthcare services and facilities in local areas.

Contributors $\mathrm{YL}$ and $\mathrm{CQ}$ conceived the study idea, analysed and interpreted the data, and prepared the manuscript. YL, A-KW, JM and CQ revised the manuscript, read the final version of the manuscript and approved the submission.

Funding The China Health and Nutrition Survey (CHNS) was supported by the China National Institute of Nutrition and Food Safety, China Centre for Disease Control and Prevention, Carolina Population Center, the University of North Carolina at Chapel Hill, the National Institutes of Health (NIH) (R01-HD30880, DK056350, R24 HD050924 and R01-HD38700) and the Fogarty International Center. YL received a grant from the Karolinska Institutet, Stockholm, Sweden (20150ste43025). CQ received grants from the Swedish Research Council (VR, 2015-2531), the Swedish Research Council for Health, Working Life and Welfare (FORTE, 2014-1382 and 2015-1352), and Karolinska Institutet, Stockholm, Sweden.

Competing interests None declared.

Patient consent Obtained.

Ethics approval The China Health and Nutrition Survey (CHNS) was approved by the Review Committees of the University of North Carolina at Chapel Hill, North Carolina, USA, and the Chinese Centre for Disease Control and Prevention, Beijing, China. All participants provided written informed consent prior to the surveys.

Provenance and peer review Not commissioned; externally peer reviewed. Data sharing statement No additional data are available.

Open Access This is an Open Access article distributed in accordance with the Creative Commons Attribution Non Commercial (CC BY-NC 4.0) license, which permits others to distribute, remix, adapt, build upon this work non-commercially, and license their derivative works on different terms, provided the original work is properly cited and the use is non-commercial. See: http://creativecommons.org/ licenses/by-nc/4.0/

(C) Article author(s) (or their employer(s) unless otherwise stated in the text of the article) 2017. All rights reserved. No commercial use is permitted unless otherwise expressly granted.

\section{REFERENCES}

1. Gill TM, Allore HG, Gahbauer EA, et al. Change in disability after hospitalization or restricted activity in older persons. JAMA 2010;304:1919-28.

2. Christensen K, Doblhammer G, Rau R, et al. Ageing populations: the challenges ahead. Lancet 2009;374:1196-208.

3. Angleman SB, Santoni G, Von Strauss E, et al. Temporal trends of functional dependence and survival among older adults from 1991 to 2010 in Sweden: toward a healthier aging. J Gerontol A Biol Sci Med Sci 2015;70:746-52.

4. Liang $Y$, Song A, Du S, et al. Trends in disability in activities of daily living among Chinese older adults, 1997-2006: the China Health and Nutrition Survey. J Gerontol A Biol Sci Med Sci 2015;70:739-45.

5. Liang $\mathrm{Y}$, Welmer AK, Wang R, et al. Trends in incidence of disability in activities of daily living in Chinese older adults: 1993-2006. J Am Geriatr Soc 2017;65:306-12.

6. Yu R, Wong M, Chang B, et al. Trends in activities of daily living disability in a large sample of community-dwelling Chinese older adults in Hong Kong: an age-period-cohort analysis. BMJ Open 2016;6:e013259.

7. Katz S, Ford AB, Moskowitz RW, et al. Studies of illness in the aged. The index of ADL: a standardized measure of biological and psychosocial function. JAMA 1963;185:914-9.

8. Lawton MP, Brody EM. Assessment of older people: selfmaintaining and instrumental activities of daily living. Gerontologist 1969;9:179-86.

9. Ward $G$, Jagger $C$, Harper W. A review of instrumental ADL assessments for use with elderly people. Rev Clin Gerontol 1998;8:65-71.

10. Hesseberg $\mathrm{K}$, Bentzen $\mathrm{H}$, Ranhoff $\mathrm{AH}$, et al. Disability in instrumental activities of daily living in elderly patients with mild cognitive impairment and Alzheimer's disease. Dement Geriatr Cogn Disord 2013;36:146-53.

11. Jekel K, Damian M, Wattmo C, et al. Mild cognitive impairment and deficits in instrumental activities of daily living: a systematic review. Alzheimers Res Ther 2015;7:17.
12. Hung WW, Ross JS, Boockvar KS, et al. Recent trends in chronic disease, impairment and disability among older adults in the United States. BMC Geriatr 2011;11:47.

13. Verropoulou G, Tsimbos C. Disability trends among older adults in ten European countries over 2004-2013, using various indicators and Survey of Health, Ageing and Retirement in Europe (SHARE) data. Ageing and Society 2016;31:1.

14. Jiang J, Tang Z, Meng XJ, et al. Demographic determinants for change in activities of daily living: a cohort study of the elderly people in Beijing. J Epidemiol 2002;12:280-6.

15. Artaud F, Dugravot A, Sabia S, et al. Unhealthy behaviours and disability in older adults: three-city Dijon cohort study. BMJ 2013;347:f4240.

16. Koyanagi A, Moneta MV, Garin N, et al. The association between obesity and severe disability among adults aged 50 or over in nine high-income, middle-income and low-income countries: a crosssectional study. BMJ Open 2015;5:e007313.

17. Welmer AK, Liang Y, Angleman S, et al. Vascular risk factor burden, atherosclerosis, and functional dependence in old age: a populationbased study. Int J Behav Med 2014;21:597-604.

18. Sousa RM, Ferri CP, Acosta D, et al. Contribution of chronic diseases to disability in elderly people in countries with low and middle incomes: a 10/66 dementia research group population-based survey. Lancet 2009;374:1821-30.

19. Rosso AL, Eaton CB, Wallace R, et al. Geriatric syndromes and incident disability in older women: results from the women's health initiative observational study. J Am Geriatr Soc 2013;61:371-9.

20. Hoeymans $\mathrm{N}$, Wong $\mathrm{A}$, van $\mathrm{Gool} \mathrm{CH}$, et al. The disabling effect of diseases: a study on trends in diseases, activity limitations, and their interrelationships. Am J Public Health 2012;102:163-70.

21. Popkin BM, Du S, Zhai F, et al. Cohort Profile: The China Health and Nutrition Survey-monitoring and understanding socioeconomic and health change in China, 1989-2011. Int J Epidemiol 2010;39:1435-40.

22. WHO. Global Recommendations on Physical Activity for Health. World Health Organization, 2010.

23. Food and Nutrition Board, Institute of Medicine of the National Academies. Dietary Reference Intakes for Energy, Carbohydrate, Fiber, Fat, Fatty Acids, Cholesterol, Protein, and Amino Acids. Washington D C: The National Academies Press, 2002/2005.

24. Zhou BF. Cooperative Meta-Analysis Group of the Working Group on Obesity in China. Predictive values of body mass index and waist circumference for risk factors of certain related diseases in Chinese adults--study on optimal cut-off points of body mass index and waist circumference in Chinese adults. Biomed Environ Sci 2002;15:83-96.

25. Chobanian AV, Bakris GL, Black HR, et al. The Seventh Report of the Joint National Committee on Prevention, Detection, Evaluation, and Treatment of High Blood Pressure: the JNC 7 report. JAMA 2003;289:2560-72.

26. Feng $Q$, Zhen Z, Gu D, et al. Trends in ADL and IADL disability in community-dwelling older adults in Shanghai, China, 1998-2008. J Gerontol B Psychol Sci Soc Sci 2013;68:476-85.

27. Freedman VA, Martin LG, Schoeni RF, et al. Declines in late-life disability: the role of early- and mid-life factors. Soc Sci Med 2008;66:1588-602.

28. Falk $\mathrm{H}$, Johansson $\mathrm{L}$, Ostling $\mathrm{S}$, et al. Functional disability and ability 75-year-olds: a comparison of two Swedish cohorts born 30 years apart. Age Ageing 2014;43:636-41.

29. Lin SF, Beck AN, Finch BK. Black-white disparity in disability among U.S. older adults: age, period, and cohort trends. J Gerontol B Psychol Sci Soc Sci 2014;69:784-97.

30. Gong P, Liang S, Carlton EJ, et al. Urbanisation and health in China. Lancet 2012;379:843-52.

31. Purser JL, Feng Q, Yi Z, et al. A new classification of function and disability in China: subtypes based on performance-based and selfreported measures. J Aging Health 2012;24:779-98.

32. Ramesh M, Wu X. Health policy reform in China: lessons from Asia Soc Sci Med 2009;68:2256-62.

33. Murabito JM, Pencina MJ, Zhu L, et al. Temporal trends in selfreported functional limitations and physical disability among the community-dwelling elderly population: the Framingham heart study. Am J Public Health 2008;98:1256-62.

34. Jeune $B$, Brønnum-Hansen $\mathrm{H}$. Trends in health expectancy at age 65 for various health indicators, 1987-2005, Denmark. Eur J Ageing 2008;5:279-85.

35. Stineman MG, Xie D, Pan Q, et al. Understanding non-performance reports for instrumental activity of daily living items in population analyses: a cross sectional study. BMC Geriatr 2016;16:64. 